\title{
Traitement superficiel d'acier par laser excimère
}

\author{
A. Pereira, A. Cros ${ }^{1}$, Ph. Delaporte, W. Marine ${ }^{1}$ et M. Sentis \\ Laboratoire Lasers, Plasmas et Procédés Photoniques, LP3, FRE 2165 du CNRS, \\ Université de la Méditerranée, Pôle Scientifique de Luminy, 163 avenue de Luminy, Case 917, \\ 13288 Marseille cedex 9, France \\ ${ }^{1}$ Groupe de Physique des États Condensés (GPEC), UMR 6631 du CNRS, \\ Université de la Méditerranée, Pôle Scientifique de Luminy, 163 avenue de Luminy, Case 901 , \\ 13288 Marseille cedex 9, France
}

\begin{abstract}
Résumé : Motivée par des nécessités économiques et environnementales, l'industrie est amenée à développer de nouveaux procédés propres, notamment pour les applications concernant la préparation de surface (nettoyage, décapage...). Dans ce domaine, l'utilisation du laser excimère présente de nombreux avantages. En effet, en raison d'un temps d'interaction de courte durée (ns), suivi d'un échauffement et d'un refroidissement rapide de la zone irradiée sur une profondeur très fine $(\mu \mathrm{m})$, il est possible de modifier localement les propriétés physico-chimiques d'une surface sans changer les propriétés intrinsèques du matériau. Le but de nos travaux a consisté en l'étude des effets d'un traitement par laser excimère $(\mathrm{XeCl})$ sur les propriétés chimiques (XPS et AES), structurales (XRD et GIXRD) et morphologiques (MEB) de surface d'acier. Outre le nettoyage de la surface, les analyses chimiques réalisées sur de l'acier ont mis en évidence pour des densités d'énergie importantes la formation d'une couche de $\mathrm{Fe}_{2} \mathrm{O}_{3}$ au détriment de $\mathrm{FeOOH}$. Les observations MEB ont révélé la création de structures sphériques nanométriques. Ce traitement permet notamment d'améliorer la tenue à la corrosion de la surface.
\end{abstract}

\section{I.INTRODUCTION}

Les techniques actuelles de préparation de surface sont souvent basées sur des procédés mécano - chimiques qui d'une part sont susceptibles d'endommager la surface et surtout génèrent une quantité importante de déchets secondaires qu'il est nécessaire de recycler. Dans certains cas, l'utilisation d'un procédé photonique permet d'obtenir des résultats équivalents, voir meilleurs, à ceux obtenus par les technologies classiques, sans créer de déchets supplémentaires. Les caractéristiques des lasers à excimères, qui sont des sources photoniques de puissance impulsionnelles émettant dans le domaine de l'ultraviolet, leur permettent d'avoir de nombreuses applications dans les domaines du nettoyage [1], du décapage de peinture, de la décontamination nucléaire $[2,3]$, de la préparation de surface avant collage, de la nitruration d'alliages métalliques [4] ou encore du traitement de surface.

Particulièrement bien adapté au nettoyage de surface, celui-ci peut s'accompagner avec l'utilisation d'une densité surfacique plus élevée de modifications superficielles des propriétés physico-chimiques du substrat. Certaines modifications peuvent avoir des effets positifs tels que l'augmentation de la dureté de la surface par un processus de nitruration [5], ou la protection contre la corrosion [6]. D'autres, par contre, comme la création de contraintes résiduelles, peuvent limiter le domaine d'utilisation du matériau traité. Il est donc particulièrement important de connaître les effets induits par le traitement laser sur les caractéristiques physiques et chimiques de la surface du matériau.

\section{DIPOSITIF EXPERIMENTAL}

Les expériences ont été menées sur des échantillons d'acier (1C) de $10 \times 10 \mathrm{~mm}^{2}$ et $3 \mathrm{~mm}$ d'épaisseur. Ils ont été irradiés sous air ambiant par le faisceau d'un laser à excimères Lambda Physics EMG $203 \mathrm{MSC}(\lambda=308 \mathrm{~nm}, \tau=25 \mathrm{~ns})$. Un système optique permettait 
d'obtenir une répartition d'énergie uniforme à la surface de l'échantillon, comprise entre 2 et $10 \mathrm{~J} / \mathrm{cm}^{2}$. Quelque soit le mode de traitement utilisé, balayage ou statique, une irradiation multi-tirs a été utilisée de façon à obtenir 10 tirs en chaque point. Une buse d'aspiration a été utilisée pour récupérer les particules ablatées.

La composition chimique de la surface a été analysée par XPS (X-ray Photoelectron Spectroscopy) afin de déterminer les liaisons chimiques sur une profondeur de $10 \mathrm{~nm}$, et par AES (Auger Electron Spectroscopy) associé à un canon à ions permettant de décaper la surface et donc de suivre l'évolution de la composition chimique en fonction de la profondeur. Des analyses ont été réalisées par GIXRD (Grazing Incidence X-Ray Diffraction) pour connaître la structure du matériau. Les observations microscopiques des surfaces irradiées ont été effectuées avec un MEB (Microscope Electronique à Balayage).

\section{ANALYSES ET COMMENTAIRES}

\subsection{Analyse chimique}

L'analyse XPS d'une surface d'acier non traitée permet d'observer des pics de $\mathrm{Fe}, \mathrm{O}, \mathrm{C}, \mathrm{Mn}$, $\mathrm{Zn}$ et $\mathrm{Ca}$. Le nettoyage de la surface réalisé par le traitement laser est mis en évidence par la disparition des pics caractéristiques de $\mathrm{Mn}, \mathrm{Zn}$ et $\mathrm{Ca}$ sur les échantillons irradiés. Lorsque la fluence laser de traitement augmente, le rapport $[\mathrm{O}] /[\mathrm{C}]$ augmente. Ceci est du à une oxydation de la surface et à l'enlèvement du carbone de pollution.

Les spectres de $\mathrm{Fe}_{2 p}$, obtenus entre 700 et $740 \mathrm{eV}$ pour différentes conditions de traitement, mettent en évidence l'apparition de Fe(III). Cette nouvelle couche d'oxyde, composée de $\mathrm{Fe}_{2} \mathrm{O}_{3}$ et de $\mathrm{FeOOH}$, a une épaisseur supérieure à $10 \mathrm{~nm}$ pour des fluences comprises entre 2 et $10 \mathrm{~J} / \mathrm{cm}^{2}$. Pour séparer les contributions respectives des deux oxydes, nous avons analysé le spectre de l'oxygène entre $525 \mathrm{et} 540 \mathrm{eV}$. La figure 1 présente le spectre d'un échantillon non traité (a) et ceux d'échantillons traités à $2 \mathrm{~J} / \mathrm{cm}^{2}$ (b) et $10 \mathrm{~J} / \mathrm{cm}^{2}$ (c). Trois pics peuvent être attribués respectivement à une liaison $\mathrm{Fe}-\mathrm{O}$ dans $\mathrm{Fe}_{2} \mathrm{O}_{3}$ et $\mathrm{FeOOH}$ $(530,4 \mathrm{eV})$, à une liaison $-\mathrm{OH}$ dans $\mathrm{FeOOH}(531,7 \mathrm{eV})$ et à l'eau $(533,3 \mathrm{eV})$. Sur les échantillons traités, la contribution de $\mathrm{H}_{2} \mathrm{O}$ disparaît et celle de $-\mathrm{OH}$ est réduite par rapport à celle de $\mathrm{Fe}-\mathrm{O}$. Cette tendance est d'autant plus marquée que la fluence est élevée. Le traitement laser induit donc une oxydation de la surface, et cette couche d'oxyde est principalement constituée de $\mathrm{Fe}_{2} \mathrm{O}_{3}$ au détriment de $\mathrm{FeOOH}$.

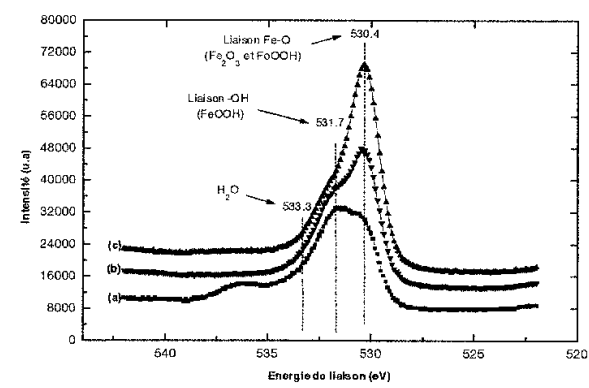

Figure 1. Spectre XPS de O1s. (a) non traité, (b) $2 \mathrm{~J} / \mathrm{cm}^{2}$, (c) $10 \mathrm{~J} / \mathrm{cm}^{2}$.

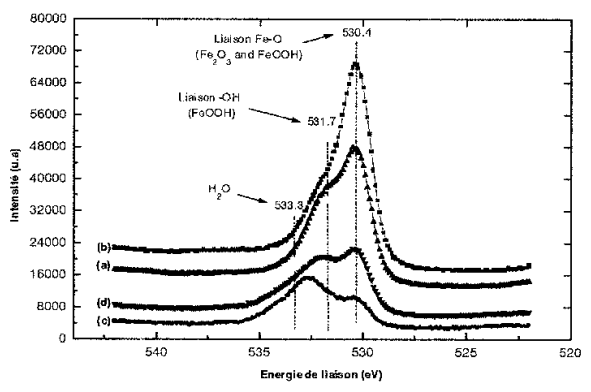

Figure 2. Evolution du spectre XPS de $\mathrm{O}_{1 \text { s }}$ en fonction du temps d'exposition à l'air après l'irradiation laser. (a) $2 \mathrm{~J} . \mathrm{cm}^{-2}$, (b) $10 \mathrm{~J} . \mathrm{cm}^{-2}$, (c) $2 \mathrm{~J} . \mathrm{cm}^{-2}$ après 1 mois, (d) $10 \mathrm{~J} . \mathrm{cm}^{-2}$ après 1 mois

La figure 2 présente l'évolution des spectres liés à l'oxygène en fonction du temps d'exposition à l'air de l'échantillon après le traitement laser. On constate qu'après un mois le pic caractéristique de $\mathrm{Fe}-\mathrm{O}$, qui est lié entre autres à la présence de $\mathrm{Fe}_{2} \mathrm{O}_{3}$ est toujours 
supérieur au pic du à la liaison $-\mathrm{OH}$ dans le cas du traitement à $10 \mathrm{~J} / \mathrm{cm}^{2}$, alors que pour l'échantillon traité à $2 \mathrm{~J} / \mathrm{cm}^{2}$, le rapport des pics est redevenu sensiblement le même que celui d'un échantillon non traité. Un traitement à forte fluence favorise donc la formation d'une couche de $\mathrm{Fe}_{2} \mathrm{O}_{3}$ qui passive la surface et augmente la résistance à la corrosion [7].

Des analyses par spectroscopie des électrons Auger ont été réalisées sur différentes zones d'un échantillon irradié sur une surface limitée par la taille du spot laser $\left(10\right.$ tirs à $\left.10 \mathrm{~J} / \mathrm{cm}^{2}\right)$. La première zone est le centre de l'impact laser, la seconde est située à l'extérieur du spot laser mais proche de son bord $(\sim 500 \mu \mathrm{m})$ et la dernière est située loin du spot laser (4 à $5 \mathrm{~mm}$ ). Les analyses ont été effectuées en utilisant un décapage ionique pour déterminer la composition chimique à différentes profondeurs. Dans l'impact laser, la surface ne présente pas de traces de carbone et est principalement constituée de $\mathrm{Fe}_{2} \mathrm{O}_{3}$. Après 900 s de décapage, on voit apparaitre le fer pur. Dans la seconde zone, proche de l'impact laser, on constate la présence d'une petite proportion de carbone qui diminue rapidement au cours du décapage. La couche d'oxyde présente à la surface est composée d'un mélange de $\mathrm{Fe}_{2} \mathrm{O}_{3}$ et de $\mathrm{FeOOH}$, et elle est plus épaisse que dans la zone irradiée car un temps de décapage de 2200 s est nécessaire pour voir apparaître de pic du Fer. Dans la dernière zone, loin de l'impact, la surface est polluée par du carbone qui empêche la détection des autres éléments. Après $120 \mathrm{~s}$ de décapage une fine couche de FeOOH apparaît, puis on retrouve le pic du fer (480s de décapage).

Ces résultats montrent que le traitement laser a pour effet de nettoyer la zone irradiée. La couche d'oxyde se forme à la surface par redépôt d'une partie des espèces ablatées sur la surface proche de l'impact laser. Le balayage de la surface par le faisceau laser permet d'obtenir ces deux effets, nettoyage et oxydation, sur la totalité de la surface.

\subsection{Analyse structurelle}

Les modifications de structure engendrées par le traitement laser ont été étudiées par des analyses par diffraction $X$ en incidence rasante $\left(\alpha=1^{\circ}\right)$. Pour un échantillon traité à $2 \mathrm{~J} / \mathrm{cm}^{2}$, on observe une légère transformation de phase $\alpha$ en phase $\gamma$, mais il n'y a pas d'apparition d'oxyde. L'acier est principalement constitué de ferrite $(\alpha-\mathrm{Fe})$ et de carbures. Lors du chauffage du à l'irradiation laser, une partie de ce matériau est transformé en austénite $(\gamma$-Fe) qui présente une structure cubique face centrée. On observe également sur les échantillons traités à $10 \mathrm{~J} / \mathrm{cm}^{2}$ cette transformation en austénite mais aussi l'apparition de phases d'oxydes. $\mathrm{FeO}$ est clairement identifié, ainsi que $\mathrm{Fe}_{2} \mathrm{O}_{3}$ et/ou $\mathrm{Fe}_{3} \mathrm{O}_{4}$.

Les analyses effectuées en incidence normale n'ont pas permis de détecter la moindre modification de structure du au traitement laser en raison d'une profondeur d'analyse trop importante $(>1 \mu \mathrm{m})$. Ceci confirme que le traitement par laser ultraviolet n'affecte qu'une fine couche à la surface du matériau irradié.

\subsection{Analyse morphologique}

La surface des échantillons a été visualisée par microscopie à balayage électronique. Nous avons à nouveau distingué deux zones : l'une dans l'impact laser et l'autre dans la proche périphérie de l'impact où les analyses XPS avaient mis en évidence la formation d'une couche de $\mathrm{Fe}_{2} \mathrm{O}_{3}$. Dans les deux zones, on observe nettement une nanostructuration de la surface. Dans l'impact laser, les particules ont un diamètre d'environ $30 \mathrm{~nm}$ et sont réparties uniformément sur la surface. La figure 3 présente une image MEB de la surface dans cette zone. Dans l'autre zone, on observe sur la photographie de la figure 4 que la surface est recouverte par des agrégats d'une centaine de nanomètres de diamètre formés par de plus petites particules $(20-30 \mathrm{~nm})$.

Lors de l'interaction entre le faisceau laser et la surface d'acier sous atmosphère ambiante, un plasma d'ablation est créé. Il contient des électrons, les espèces présentes à la surface dans des états neutres et ionisés, et les composés du gaz de couverture, en l'occurrence l'air. Dans ce plasma, de nombreuses réactions se produisent et de nouvelles 
molécules se forment [8]. Les conditions de température et de pression favorisent notamment l'apparition de $\mathrm{Fe}_{2} \mathrm{O}_{3}$. Les particules qui ne sont pas aspirées par la buse se redéposent alors sur la surface en se refroidissant et en formant des agrégats [9].

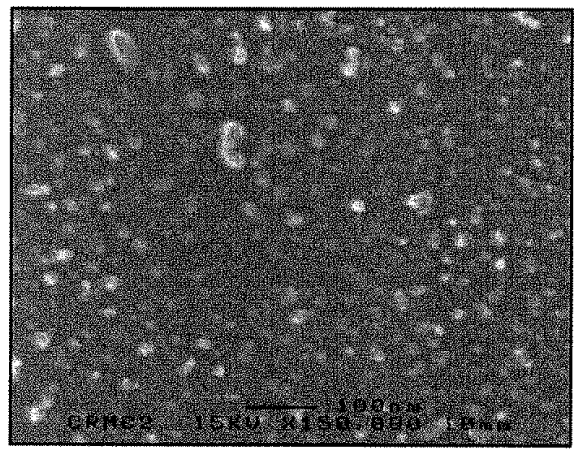

Figure 3. Image MEB de la surface au centre de l'impact laser

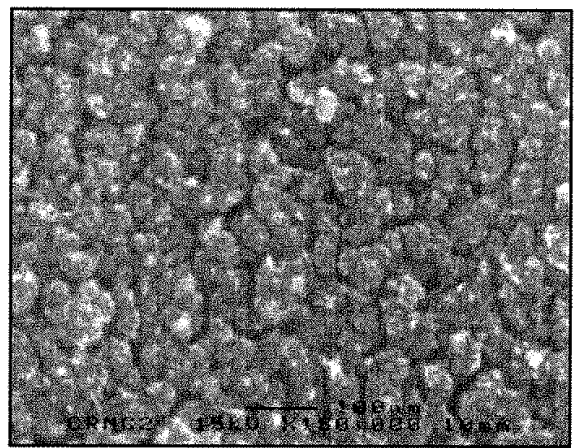

Figure 4. Image MEB de la surface à la proche périphérie de l'impact laser

\section{CONCLUSION}

Les analyses effectuées sur des surfaces d'acier irradiées par le faisceau d'un laser à excimères montrent que le traitement induit la formation d'oxyde de $\mathrm{Fe}$ (III), principalement $\mathrm{Fe}_{2} \mathrm{O}_{3}$. L'épaisseur de la couche d'oxyde est de quelques dizaines de nanomètres. L'augmentation de la fluence laser favorise la formation de $\mathrm{Fe}_{2} \mathrm{O}_{3}$ au détriment de $\mathrm{FeOOH}$ et génère une épaisseur d'oxyde plus importante. La fusion d'une fine couche superficielle d'acier provoque la transformation de $\alpha-\mathrm{Fe}$ en $\gamma$-Fe, et donc un changement de phase dans cette couche ainsi que l'apparition de phases oxydes. Lors d'une précédente étude [10], nous avons montré qu'une fluence de l'ordre de $2.6 \mathrm{~J} / \mathrm{cm}^{2}$ permettait d'atteindre la température de vaporisation $\left(3823 \mathrm{~K}\right.$ ) de cet acier. A $10 \mathrm{~J} / \mathrm{cm}^{2}$, un plasma significatif est donc crée, avec une température à la surface supérieure aux températures de fusion du fer $(1808 \mathrm{~K})$ et des différents oxydes de fer ( $\mathrm{FeO}: 1693 \mathrm{~K}$ et $\mathrm{Fe}_{2} \mathrm{O}_{3}: 1838 \mathrm{~K}$ ). Ainsi, pendant la durée de l'état liquide, l'oxygène pénètre dans le bain liquide, diffuse et pendant la solidification réagit pour former différentes phases oxyde du fait de cinétiques différentes. Lors de nos traitements multi-tirs, chaque tir permet l'introduction d'oxygène dans le substrat pour former des phases oxydes et dans le même temps chaque tir fond les oxydes précédemment formés. On a alors une augmentation de l'épaisseur d'oxyde. La couche d'oxyde formée dans le bain liquide est complétée par une couche de $\mathrm{Fe}_{2} \mathrm{O}_{3}$ formée par redépôt des particules ablatées à la périphérie du spot laser. On observe une nanostructuration de la surface qui est constituée d'agrégats de molécules de $\mathrm{Fe}_{2} \mathrm{O}_{3}$. Cette couche passive la surface et la protège contre la corrosion, et augmente la surface équivalente de celle-ci.

\section{Références}

[1] P. Maravelaki-Kalaitzaki, V. Zafiropulos, C. Fotakis. Appl. Surf. Sci. 148, (1999) 92.

[2] Sentis M., Delaporte Ph., Marine W., Uteza O., Quantum. Electron., 30 (2000) 495-500.

[3] Delaporte Ph., Gastaud M., Marine W., Sentis M., Uteza O., Thouvenot P., Alcaraz J.L., Le Samedy J.M., Blin D., ., Appl. Surf. Sci., 197-198 (2002) 826-830.

[4] Schaaf P., Prog. Mater. Sci., 47 (2002) 1-161. 
[5] Sicard E., Andreazza-Vignolle C., Boulmer-Leborgne C., Delaporte Ph., Sentis M., Frainais M., Proc. SPIE 3885 (2000) 148-158.

[6] Copola C., Avram I., Terzzoli M., Duhalde S., Morales C., Perez T., Audebert F., Delaporte Ph., Sentis M., Appl. Surf. Sci., 197-198 (2002) 896-903.

[7] Aggudelo A., Gancedo J., Marco J., Creus M., Gallego-Lluesma E., Desimoni J., Mercader R., Appl. Surf. Sci., 148 (1999) 171-182.

[8] Itina T., Hermann J., Delaporte Ph., Sentis M., Phys. Rev. E (2002) publication acceptée.

[9] Marine W. Patrone L., Luk'yanchuk B., Sentis M., Appl. Surf. Sci., 154-155 (2000) 345352.

[10] A. Pereira, A. Cros, Ph. Delaporte, W. Marine, M. Sentis, Appl. Phys. A (2002), accepté. 